Marquette University

e-Publications@Marquette

Mathematics, Statistics and Computer Science

Mathematics, Statistics and Computer Science,

Faculty Research and Publications

Department of

$2-1-2014$

\title{
Quantifying the Statistical Impact of GRAPPA in fcMRI Data with a Real-Valued Isomorphism
}

Iain P. Bruce

Marquette University

Daniel B. Rowe

Marquette University, daniel.rowe@marquette.edu

Accepted version. IEEE Transactions on Medical Imaging, Vol. 33, No. 2 (February 2014): 495-503.

DOI. (C) 2014 Institute of Electrical and Electronics Engineers (IEEE). Used with permission. 


\title{
Quantifying the Statistical Impact of GRAPPA in fcMRI Data With a Real-Valued Isomorphism
}

\author{
Iain P. Bruce \\ Department Mathematics, Statistics and Computer Science, Marquette University, \\ Milwaukee, WI \\ Daniel B. Rowe \\ Department Mathematics, Statistics and Computer Science, Marquette University, \\ Milwaukee, WI
}

\begin{abstract}
The interpolation of missing spatial frequencies through the generalized auto-calibrating partially parallel acquisitions (GRAPPA) parallel magnetic resonance imaging (MRI) model implies a correlation is induced between the acquired and reconstructed frequency measurements. As the parallel image reconstruction algorithms in many medical MRI scanners are based on the GRAPPA model, this study aims to quantify the statistical implications that the GRAPPA model has in functional connectivity studies. The linear mathematical framework derived in the work of Rowe, 2007, is adapted to represent the complex-valued GRAPPA image reconstruction operation in terms of a real-valued isomorphism, and a statistical analysis is performed on the effects that the GRAPPA operation has on reconstructed voxel means and correlations. The interpolation of missing spatial frequencies with the GRAPPA model is shown to result in an artificial correlation induced between voxels in the reconstructed images, and these artificial correlations are shown to reside in the low temporal frequency spectrum commonly associated with functional connectivity. Through a real-valued isomorphism, such as the one outlined in this manuscript, the exact artificial correlations induced by the GRAPPA model are not simply estimated, as they would be with simulations, but are precisely quantified. If these correlations are unaccounted for, they can incur an increase in false positives in functional connectivity studies.
\end{abstract}


NOT THE PUBLISHED VERSION; this is the author's final, peer-reviewed manuscript. The published version may be accessed by following the link in the citation at the bottom of the page.

\section{SECTION I.}

\section{Introduction}

Amagnetic resonance imaging (MRI) scanners inability to instantly acquire the spatial frequency spectrum of an object being scanned imposes constraints on the spatial and temporal resolution of images acquired in echo planar imaging schemes, such as those used in functional connectivity MRI (fcMRI). Since postulated in, ${ }^{1}$ parallel MRI (pMRI) has become one of the most prevalent areas of studies that aim to improve the spatial and/or temporal resolution of fcMRI images. The basis of pMRI is such that the complex-valued spatial frequency spectrum ( $k$-space) can be acquired from an array of receiver coils concurrently, and then combined into a single image using pMRI techniques such as SENSitivity Encoding (SENSE) ${ }^{2}$ and generalized auto-calibrating partially parallel acquisitions (GRAPPA). ${ }^{3}$ Taking advantage of the overlap of spatial information from each receiver coil, $k$-space can be sub-sampled by a factor of $A$ by acquiring only every $A^{\text {th }}$ line of spatial frequencies in the phase encoding (PE) direction, resulting in reduced field-of-view (FOV) aliased images in the image domain.

Recently, Bruce et al.4-5,6,7 demonstrated that the "unfolding" of aliased coil images into a single full FOV combined image with the SENSE model induces a previously unquantified artificial correlation between unaliased voxels. As the GRAPPA model interpolates missing $k$-space values from acquired measurements both within a coil and between coils, the operation by definition induces a local correlation between the interpolated and acquired spatial frequencies. Based on the properties of the inverse Fourier transform (IFT) used to convert coil spatial frequencies into coil images, any operation that induces a local correlation between individual frequencies in $k$-space implies a global correlation between voxels in the image domain. This induced correlation must therefore be explored and precisely quantified.

When a real-valued object is placed in an MRI scanner, magnetic field (B-field) gradients Fourier encode $k$-space to acquire complex-valued frequency measurements with both real and imaginary components. If a further phase distortion is invoked by factors such as B-field inhomogeneities (that can arise from respiration, improper field shimming, etc.), transverse relaxation, and chemical shifts, ${ }^{8-9,10,11}$ the conjugate Hermitian symmetry of $k$-space is broken, which results in IFT reconstructed voxel values being complex-valued as well. In recent studies, ${ }^{12-13,14,15,16}$ it has been shown that models that employ both a magnitude and phase time series have a greater statistical power in detecting functional activations in the brain than traditional magnitude-only models. Furthermore, if spatial 
processing and image reconstruction operations are applied to complex-valued data, an artificial correlation is induced between the real reconstructed voxel values, between the imaginary reconstructed voxel values, and between the real and imaginary (real/imaginary) reconstructed voxel values. ${ }^{4-5,6,7,17,18}$ The real, imaginary and real/imaginary correlations cannot be precisely distinguished when the operations are represented in a complex-valued form and performed on a voxel-by-voxel basis. This provides the motivation for using a real-valued isomorphism, in which the complex-valued operation is performed on all voxels at once using mathematically equivalent real-valued matrix operators, allowing for all correlations induced by that process to be quantified.

In this manuscript, we further develop the linear AMMUST (A Mathematical Model for Understanding the STatistical) framework, ${ }^{14,15}$ to perform a mathematically equivalent application of the conventional complex-valued GRAPPA image reconstruction to all subsampled spatial frequencies from an array of receiver coils at once using a real-valued linear isomorphism. To create such an isomorphism, the individual operations involved in carrying out the GRAPPA reconstruction process are presented in terms of real-valued matrices that permute and interpolate the real and imaginary missing lines of $k$-space. An isomorphism of this kind provides a means of deducing the correlations artificially induced by the GRAPPA model both precisely and directly, without the need for time consuming approximations through Monte Carlo simulations. ${ }^{20,21}$ While the GRAPPA operation has been previously represented in terms of a complex-valued matrix operator, ${ }^{22,23}$ the correlations induced between the real, imaginary and real/imaginary reconstructed voxels cannot be determined from that formulization. The correlations induced by the GRAPPA operators are theoretically illustrated and simulated on phantom data as well as on experimental human subject fcMRI data. It is shown in both theoretical and experimental illustrations that the correlations induced by the GRAPPA operation are still present after band-pass filtering voxel time series to the $<0.08 \mathrm{~Hz}$ frequency band commonly observed in fcMRI studies. ${ }^{24}$ This suggests that the induced correlations can have null hypothesis fcMRI implications, where a zero correlation is assumed between voxels.

\section{SECTION II.}

\section{Theory}

\section{A. The GRAPPA Model}

As illustrated in Fig. 1, the GRAPPA reconstruction generates a full FOV uncombined spatial frequency array for each receiver coil. When sub-sampling occurs in the PE 
direction, an interpolation kernel is used to fit the acquired data from $N_{\text {col }}$ columns and $N_{\text {row }}$ rows in all $N_{C}$ receiver coils, $F_{l}$, where the acquired rows are spaced $A \Delta k_{y}$ apart, to the $(A-1)$ additionally acquired calibration signal measurements, $F_{\text {calib }}$, in a single coil, $j$. A set of kernel weights, $w$, can be estimated and used to generate the missing spatial frequencies for each coil $j=\left[1,2, \ldots N_{C}\right]$ by solving

$$
\begin{aligned}
& F_{\text {calib }, j}\left(k_{x}, k_{y}+m \Delta k_{y}\right) \\
& =\sum_{l=1}^{N_{C}} \sum_{c=-L}^{R} \sum_{r=-U}^{D} w(j, c, r, l, m) F_{l}\left(k_{x}+c \Delta k_{x}, k_{y}+r A \Delta k_{y}\right) .
\end{aligned}
$$

In (1), $m$ denotes the $k$-space row number, $m \Delta k_{y}$ denotes the frequency offset from the acquired frequency in the $k_{y}$ direction at position $\left(k_{x}, k_{y}\right)$, the number of rows above $(U)$ and below $(D) F_{\text {calib }}$ sum to a total of $N_{\text {row }}$ rows, and the number of columns to the left $(L)$ and right $(R)$ of $F_{\text {calib }}$ sum to a total of $N_{\text {col }}$ columns in the interpolation kernel. While more recent adaptations of the GRAPPA model employ a 2-D interpolation kernel, ${ }^{25-26,27,28}$ (1) simplifies to the original 1-D interpolation derived in [3] when $c$ is set to zero.

Repeating this process for all coils results in a complete array of spatial frequencies for each coil, which if combined in either the image or frequency domains produces a full FOV reconstructed image after an IFT.

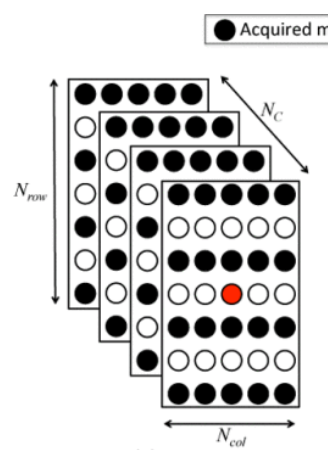

(a)

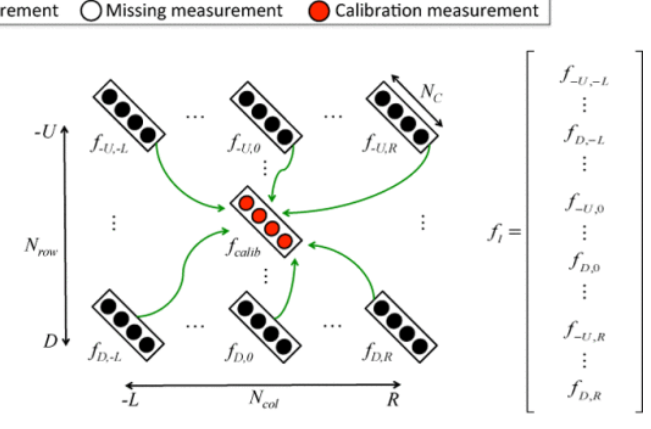

(b)

Fig. 1. The (a) $N_{\text {row }}$ rows and $N_{\text {col }}$ columns in $N_{C}$ coils, (b) used in the GRAPPA model in (2), vectorized to form $f_{l}$ in (4). 


\section{B. GRAPPA Interpolation Weights}

Interpolation weights are derived from training data with a sufficient SNR through either additionally acquired auto-calibration signal (ACS) lines in the center of $k$-space, or through full FOV pre-calibration scans. ${ }^{29}$ Although ACS lines can be used for both estimating weights and incorporated into the final reconstructed image, this approach results in data with varying effective inner-echo spacing if acquired using an EPI pulse sequence. As such, a uniform sub-sampling scheme is used in this study, with interpolation weights in (1) estimated from full FOV calibration scans.

The weights used in the GRAPPA interpolation are considered shift invariant, 3,30 and the contribution from each acquired measurement is relative to its distance from the block of $(A-1)$ missing measurements to be interpolated. Illustrated in Fig. 1, for an application of (1) to an array of $N_{C}$ coils using a kernel of size $N_{\text {row }}$ rows by $N_{\text {col }}$ columns, sub-sampled with an acceleration factor of $A=2$, (1) can be applied for all coils at once using a matrix representation by

$$
f_{\text {calib }}=w_{f l}
$$

In (2) $f_{\text {calib }}$ is a $(A-1) N_{C} \times$ column vector with $N_{C}$ sub-vectors of the $(A-1)$ complex-valued calibration spatial frequency values from each coil. In this manuscript, a column-wise application of (1) is employed. As shown in Fig. 1(b), the vector $f_{l}$ in (2) is constructed by first stacking the complex-valued acquired spatial frequencies from the $N_{C}$ coils into vectors. Moving through the kernel from top to bottom and left to right, the $N_{\text {row }}$ vectors of length $N_{C}$ in each column are stacked into $N_{\text {col }}$ vectors, which are in turn stacked into a single vector of length $N_{\text {row }} N_{\text {col }} N_{C} \times$. Using a least squares estimation, one can solve for the $(A-1) N_{C} \times N_{\text {row }} N_{\text {col }} N_{C}$ matrix of complex-valued weights in (2) by

$$
w=f_{\text {calib }} f_{l}^{H}\left(f_{l} f_{l}^{H}\right)^{-1} \text {. }
$$

To account for noise in the acquired data, one shifts through the calibration data using the technique outlined in, ${ }^{31}$ and stacks the vectors $f_{\text {calib }}$ and $f_{l}$ in (2), from at least $N_{\text {fits }} \geq N_{\text {row }} N_{\text {col }} N_{C}(A-1)$ fits, into the columns of matrices $F_{\text {calib }}=\left[f_{\text {calib }, 1}, \ldots f_{\text {calib }, N \text { fits }}\right]$ and $F_{l}=\left[f_{l, 1}, \ldots f_{l, N \text { fits }}\right]$, respectively. This allows for the interpolation weights to be determined by

IEEE Transactions on Medical Imaging, Vol 33, No. 2 (February 2014): 495-503. DOI. This article is (C) Institute of Electrical and Electronics Engineers (IEEE) and permission has been granted for this version to appear in e-Publications@Marquette. Institute of Electrical and Electronics Engineers (IEEE) does not grant permission for this article to be further copied/distributed or hosted elsewhere without the express permission from Institute of Electrical and Electronics Engineers (IEEE). 


$$
w=F_{\text {calib }} F_{l}^{H}\left(F_{l} F_{l}^{H}\right)^{-1} \text {. }
$$

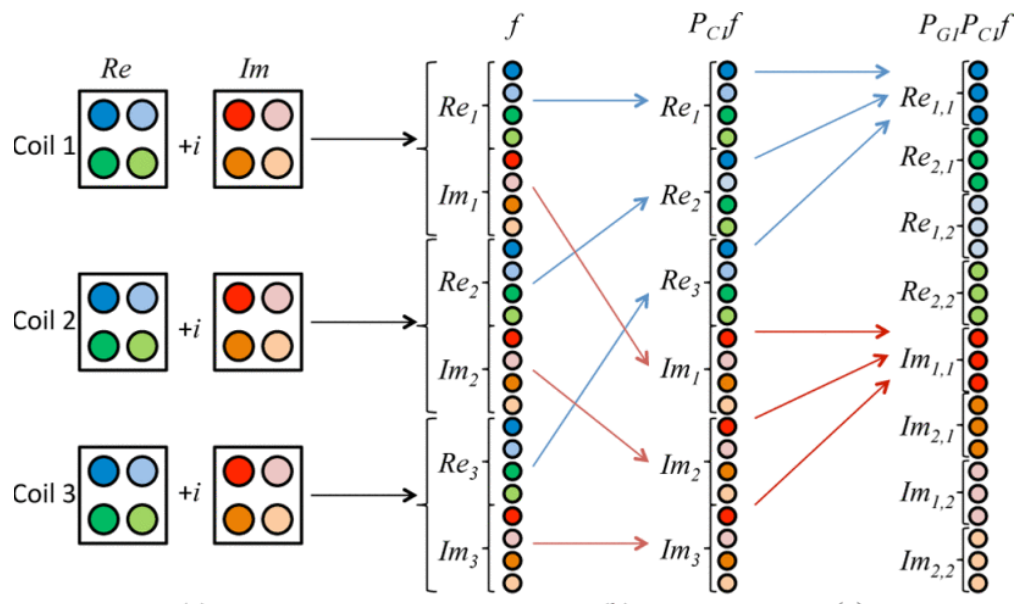

(a) (b) (c)

Fig. 2. (a) The vectorization of $N C=3$ coils into a single vector, $f$, (b) permuted via complex permutation, $P_{C 1}$, and (c) permuted by $P_{G 1}$ for application of the real-valued GRAPPA operator.

\section{Linear Framework}

The GRAPPA interpolation in (1) effectively performs a sensitivity encoding 2 in $k$ space through a convolution between sub-sampled spatial frequency measurements and the Fourier transform (FT) of the B-field sensitivities of each receiver coil. By definition, an interpolation induces a local correlation between neighboring spatial frequencies, and this correlation becomes global between voxels after an IFT. Furthermore, as the convolution kernel is derived from the FT of coil sensitivities, this correlation is directly related to the spatial localization used for unaliasing voxels, and is therefore expected to be greatest between previously aliased voxels. To observe this correlation, a mathematically equivalent representation of the GRAPPA model is presented to interpolate all missing complex-valued spatial frequencies in all coils at once using real-valued matrix operators.

The weights in (4) are described for an interpolation with data ordered by column, and thus the sub-sampled acquired data needs to be appropriately formatted. For a $p_{x} \times p_{y} / A \times N_{C}$ array of complex-valued sub-sampled spatial frequencies in multiple receiver coils, illustrated in Fig. 2(a) for a "toy" example with $N_{C}=3$ coils, a real-valued vector, $f$, is generated by stacking the rows of the real and imaginary measurements of each coil into separate vectors, stacking the vector of real values on top of the corresponding vector of imaginary values for each coil, and finally concatenating the 
resulting $N_{C}$ vectors into a single $2 N_{C} p_{x} p_{y} / A \times$ vector. A complex permutation, $P_{C 1}$, is then applied to reorder $f$ to having all real spatial frequencies from all $N_{C}$ coils stacked upon all imaginary spatial frequencies from all $N_{C}$ coils, as illustrated in Fig. 2(b). The vector of subsampled spatial frequencies in Fig. 2(b) is permuted again such that the resulting vector

$$
f_{G}=P_{G 1} P_{C 1} f
$$

has spatial frequencies ordered first by column, then by row, and finally by coil, as illustrated in Fig. 2(c).

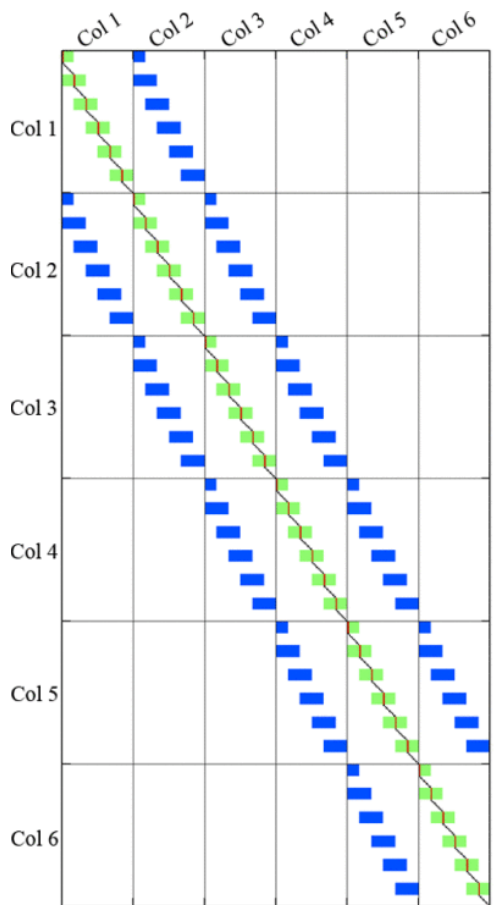

(a)

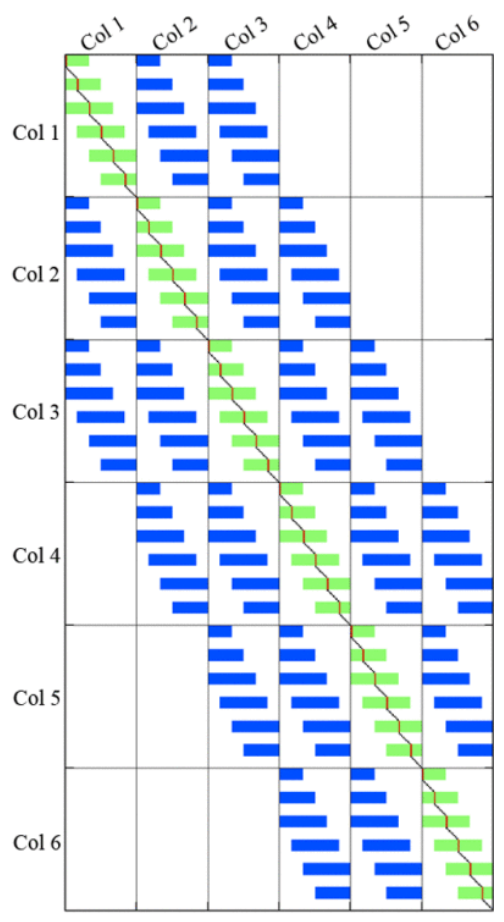

(b)

Fig. 3. Complex-valued GRAPPA matrix operators for interpolation kernels of size (a) $2 \times 1$ (green) and $2 \times 3$ (green and blue), (b) $4 \times 1$ (green) and $4 \times 5$ (green and blue). GRAPPA model is performed on a $6 \times 12$ FOV, sub-sampled by $A=2$ with $N_{C}=4$ coils.

In Fig. 3, the "toy" example from Fig. 2 is expanded to having $p_{x}=6$ columns, $p_{y}=$ 12 rows, sub-sampled by $A=2$ in $N_{C}=4$ coils. For each block of $(A-1) N_{C}$ missing spatial frequencies, the complex-valued weights in (4) are positioned such that the appropriate columns of acquired measurements are employed in the interpolation. For a complexvalued matrix implementation of GRAPPA, the layout for the $N_{\text {row }} \times N_{\text {col }}$ kernels of size $2 \times 3$ and $4 \times 5$ are presented in Fig. 3(a) and (b), respectively. The weights for each 
interpolation are segmented to incorporate the appropriate rows in the appropriate columns for the respective kernel size. In both Fig. 3(a) and (b), the green portion of the weights represents 1 -D kernels of size $2 \times 1$ and $4 \times 1$, respectively, while the combination of green and blue comprise the complete 2-D kernels. In each block of weights, the vertical red line in the green portion denotes the center of the kernel. While one could utilize a variety of techniques to refill the measurements near the edge of $k$-space (zero-filling, symmetry, wraparound, etc.), partial kernels were used in this study for simplicity, with weights, specific for each edge condition assuming no wraparound, estimated using (4). To maintain all acquired lines in the refilled array, $N_{C} \times N_{C}$ real-valued identity matrices are placed between the blocks of weights. For the complex-valued GRAPPA matrix operator, $G_{C}=\left(G_{R}+i G_{I}\right)$, in Fig. 3 to be applied to the permuted $f_{G}$ vector in (5), a real-valued representation is derived by

$$
G=\left[\begin{array}{cc}
G_{R} & -G_{I} \\
G_{I} & G_{R}
\end{array}\right]
$$

where $G_{R}$ and $G_{I}$ are the real and imaginary parts of $G_{C}$.

After applying the GRAPPA operator, $G$ in (6), to the permuted vector in (5), the refilled vector of spatial frequencies is re-permuted using the reverse of the operations in $P_{C 1}$ and $P_{G 1}$ with permutations $P_{C 2}$ and $P_{G 2}$, respectively, which are of a larger dimension. This results in a $2 N_{C} p_{x} p_{y} \times$ vector with full FOV spatial frequencies, $f$ full, with vectors of the real values for each coil, vectorized by row, stacked upon the imaginary values for the corresponding coil

$$
f_{\text {full }}=P_{C 2} P_{G 2} G f_{G}
$$

in an order similar to that of vector $f$ in Fig. 2(a). To reconstruct a vector of spatial frequencies into voxel values, the real-valued matrix representation of the complex-valued IFT, $\Omega$, was derived by Rowe et al. ${ }^{19}$ At this stage, one can either reconstruct each coil image, using a Kronecker product, $\left(I_{N_{C}} \otimes \Omega\right)$, and apply a combination matrix, $C$, to perform the combination in the image domain

$$
y=C\left(I_{N_{C}} \otimes \Omega\right) f_{\text {full }}
$$


or, to reduce the demand on computational resources, combine the coil frequencies in $k$ space and inverse Fourier reconstruct to get a single combined image

$$
y=\Omega C f_{\text {full }}
$$

While not in the scope of this study, the $\Omega$ operator in (7) and (8) can be adjusted to account for intra-acquisition decay $\left(T_{2}^{*}\right)$ and B-field inhomogeneities acquired in the $k$ space signal ${ }^{8,18}$ if $T_{2}^{*}$ or $\Delta B$ maps can be obtained. As the traditional magnitude reconstruction is not linear in nature, it is not used in this manuscript. In most studies, a magnitude reconstruction is used and the phase is discarded, ${ }^{3}$ however it has been shown in 14,32-33,34 that there is important biological information that can be obtained from the phase. The combination matrix in this manuscript, $C$, therefore performs a complex linear combination, generating a $2 p_{x} p_{y} \times$ vector of the reconstructed image containing subvectors for the real and imaginary parts as in (7), or of the combined spatial frequencies in (8). While a root sum of squares combination might be more commonly used, the process was not adopted in this study, as it is not linear.

\section{GRAPPA Operator-Induced Correlations}

The complete set of matrix operators used in the GRAPPA image reconstruction isomorphism is

$$
O_{G}=\Omega C P_{C 2} P_{G 2} G P_{G 1} P_{C 1}
$$

If the vector of observed $k$-space measurements has a mean, $f_{0}$, and a covariance, $\Gamma$, which can include phase distortions such as B-field inhomogeneities, transverse relaxation, and chemical shifts, then the reconstructed image vector, $y$, will have a mean of $\mu_{G}=$ $\mathrm{E}[y]=O_{G} f_{0}$, and a covariance of $\Sigma_{G}=\operatorname{cov}(y)=O_{G} \Gamma O_{G}{ }^{T}$, or

$$
\Sigma_{G}=\Omega C P_{C 2} P_{G 2} G P_{G 1} P_{C 1} \Gamma P_{C 1}{ }^{T} P_{G 1}{ }^{T} G^{T} P_{G 2}^{T} P_{C 2}^{T} C^{T} \Omega^{T}
$$

Since all of the permutations in (9) simply rearrange the data that they pre-multiply, they are orthonormal and do not induce any covariance, but rather rearrange any preexisting covariance in $\Gamma$. The GRAPPA operator, $G$, from (6) is not orthogonal, producing 
an identity matrix scaled by a constant, $G G^{T} \neq k_{G} I$ and therefore induces a nonidentity covariance. Although the permutations, $P_{C 2}$ and $P_{G 2}$, and the IFT operator, $\Omega$, are orthogonal, since the GRAPPA operator that they pre-multiply is not orthogonal, the resulting covariance in (10) will not be identity, even if the covariance of the original data, $\Gamma$, was assumed to be identity.

The correlation induced between voxels by the GRAPPA image reconstruction operators is found by

$$
\operatorname{corr}\left(O_{G} \Gamma O_{G}^{T}\right)=D_{0}^{-1 / 2} O_{G} \Gamma O_{G}^{T} D_{0}^{-1 / 2}
$$

where $D_{0}=\operatorname{diag}\left(O_{G} \Gamma O_{G}^{T}\right)$ is a diagonal matrix of the variances drawn from the diagonal of the covariance matrix $O_{G} \Gamma O_{G}^{T}$. The correlation induced solely by the GRAPPA image reconstruction process is deduced by assuming an identity covariance structure in the data, $\Gamma=I$, reducing $(11)$ to

$$
\operatorname{corr}\left(O_{G} O_{G}^{T}\right)=D_{0}^{-1 / 2} O_{G} O_{G}^{T} D_{0}^{-1 / 2}
$$

The real-valued isomorphism correlation matrices produced by (11) and (12) can be partitioned into $p_{x} p_{y} \times p_{x} p_{y}$ quadrants as

$$
\operatorname{corr}\left(\Sigma_{G}\right)=\left[\begin{array}{cc}
R R & R I \\
I R & I I
\end{array}\right]
$$

where quadrant $R R$ denotes the correlation between the real voxel values, quadrant $I I$ denotes the correlation between the imaginary voxel values, and quadrant $R I$ denotes the correlation between the real and imaginary voxel values. The correlation between a voxel $r$ and all other voxels in the reconstructed image can be generated by partitioning the $r$ th row of each quadrant in $\operatorname{corr}\left(\Sigma_{G}\right)$ into $p_{x}$ vectors of $\times p_{y}$, stacking these row vectors into a matrix, and transposing. 
NOT THE PUBLISHED VERSION; this is the author's final, peer-reviewed manuscript. The published version may be accessed by following the link in the citation at the bottom of the page.

\section{SECTION III.}

\section{Methods}

\section{A. Theoretical Illustration}

To theoretically illustrate the correlations induced by the GRAPPA model, both full FOV and sub-sampled time series of 490 images were generated for each of $N_{C}=8$ coils using the MR signal equation

$$
\begin{aligned}
& f\left(k_{x}, k_{y}, t\right) \\
& =\iint \rho(x, y) e^{-t / T_{2}^{*}(x, y)-i \gamma B(x, y) t-i 2 \pi\left(k_{x} x+k_{y} y\right)} d x d y \text {. }
\end{aligned}
$$

For the proton spin density, $\rho$, a 96×96 Shepp-Logan phantom was used with a simulated $T_{2}^{*}$ of $49 \mathrm{~ms}$ for white matter, $42 \mathrm{~ms}$ for grey matter, $2200 \mathrm{~ms}$ for CSF, and set to $10^{-6} \mathrm{~ms}$ in space. The B-fields, $B$, for each coil were estimated from experimentally acquired human subject data by fitting a third order polynomial to the estimated coil sensitivities from the experimental human data. Independent and identically distributed (IID) mean 0 and variance of 1 Gaussian noise was added to both components each of 490 images in a time series for each coil. The quality of GRAPPA reconstructed images is known to depend on both the kernel size and choice of $A .35,36$ Weights were therefore estimated from full FOV data to form $G$ in (6) and (9) for kernels of size $4 \times 1,2 \times 3$, and $4 \times 5$ with $A=2$ and $A=3$, to explore the effects that each parameter has on theoretical GRAPPA induced correlations.

For a Monte Carlo (MC) simulation of the GRAPPA induced correlations, an additional accelerated data set was generated with the MR signal equation. Rather than sub-sampling retrospectively, sub-sampling was simulated in the anterior-posterior PE direction by shifting through $k$-space in increments of $A \Delta k_{y}$, with an acceleration factor of $A=2$, as commonly used in fcMRI. Using the full FOV data for calibration, the accelerated data set was reconstructed using the GRAPPA model with a $4 \times 5$ interpolation kernel. After inverse Fourier reconstruction, the full FOV calibration data set was averaged over coils into a single time series to provide a baseline for comparison with the GRAPPA reconstructed time series. An average was used in place of a root sum of squares combination to match the framework in (7) and (8) as the process is linear. In fcMRI studies, voxel time series are band-pass filtered to observe frequencies below $0.08 \mathrm{~Hz}$ in an 
effort to eliminate BOLD signal changes correlated with physiological effects such as respiration, ${ }^{37}$ and observe fluctuations in the BOLD signal in the nontask condition that demonstrate patterns of coherence with specific networks in the brain. ${ }^{38}$ To observe the degree to which the GRAPPA induced correlations affect fcMRI statistics, voxel time series were convolved with a temporal Hamming band-pass filter ${ }^{39}$ with cutoff frequencies set to 0.009 and $0.08 \mathrm{~Hz}{ }^{24}$ after which both the full FOV and GRAPPA reconstructed time series were spatially filtered using a Gaussian smoothing kernel with a full-width at halfmaximum of two voxels.

\section{B. Experimental Illustration}

To observe the degree to which the statistical implications of the GRAPPA model manifest in experimental data, two nontask human subject fcMRI data sets were acquired from an array of eight receiver coils in a 3.0T General Electric Signa LX magnetic resonance imager. The first data set was composed of nine $2.5-\mathrm{mm}$-thick axial slices that are $96 \times 96$ in dimension for a $24 \mathrm{~cm} \mathrm{FOV}$, with the PE direction oriented as anterior to posterior (bottom-top in images), while the second data set was acquired under the same conditions with the PE dimension sub-sampled by $A=2$. Acquired for a series of $510 \mathrm{TRs}$, both acquired data sets had a repetition time (TR) of $1 \mathrm{~s}$, an echo time of $45.4 \mathrm{~ms}$, an effective echo spacing of $816 \mu \mathrm{s}$, a flip angle of $45^{\circ}$, and an acquisition bandwidth of $125 \mathrm{kHz}$. The first 20 TRs were discarded to account for $\mathrm{T}_{1}$ effects and varying echo times, resulting in 490 TRs acquired under the same conditions. Data was acquired with an echo planar imaging (EPI) pulse sequence and reconstructed using locally developed software. The center row of $k$-space for each receiver coil was acquired with three navigator echoes in order to estimate the error in the center frequency and group delay offsets between the odd and even $k$-space lines. ${ }^{40}$

\section{Analysis}

To observe the effects of the GRAPPA reconstruction process on the correlation structure of each data set, both theoretical and MC simulated correlations are presented between the real parts, between the imaginary parts, and between the real and imaginary (real/imaginary) parts of the reconstructed voxel values. Additionally, correlations for magnitude-squared data are presented in lieu of the correlations for magnitude only data, because the correlation structure of magnitude-squared data is asymptotically equivalent and visually similar to the correlations of magnitude data, yet magnitude-squared correlations are linear in nature when magnitude correlations are not. ${ }^{18,41}$ The null hypothesis in an fcMRI study assumes that no correlation exists between voxels, and thus 
any voxels that exhibit a statistically significant correlation in reconstructed data are deemed functionally connected. As the theoretical data was generated with no inherent correlation structure between voxels, seed voxels in both the theoretical and MC simulations were selected in regions of the phantom that were aliased prior to GRAPPA reconstruction. In the experimental data, the seed voxel was selected in a region of the brain that was both aliased prior to a GRAPPA reconstruction of sub-sampled data, and exhibited no correlation in full FOV data with a voxel in the location that would have been previously aliased had sub-sampling been performed.

Correlations in each illustration are presented about the seed voxel in three different ways. First, theoretical correlations induced solely by the GRAPPA reconstruction operators are found by assuming an identity covariance structure, $\Gamma=I$, as in (12). Second, simulated correlations are estimated directly from the reconstructed MC simulation in the theoretical illustration, and from the reconstructed experimental time series for the human subject. Third, the correlation coefficient about the seed voxel in each time series is presented after the reconstructed voxel time series are Hamming band-pass filtered to 0.009 and $0.08 \mathrm{~Hz}$.

Spatial filtering has become a common practice in many fMRI and fcMRI studies, ${ }^{42-}$ 43,44 and the effects of spatial filtering have recently been shown to exaggerate the correlations induced by image reconstruction processes. ${ }^{4-5,6,7,18}$ As such, all images in the reconstructed time series have been spatially filtered by a Gaussian smoothing kernel operator, $S$, which when added to the GRAPPA reconstruction operators in (9)

$$
S O_{G}=S \Omega C P_{C_{2}} P_{G_{2}} G P_{G_{1}} P_{C_{1}}
$$

results in spatially filtered images with a covariance of

$$
S \Sigma_{G} S^{T}=S \Omega C P_{C 2} P_{G 2} G \Gamma G^{T} P_{G 2}^{T} P_{C 2}^{T} C^{T} \Omega^{T} S^{T}
$$


NOT THE PUBLISHED VERSION; this is the author's final, peer-reviewed manuscript. The published version may be accessed by following the link in the citation at the bottom of the page.

\section{SECTION IV.}

\section{Results}

\section{A. Theoretical Results}

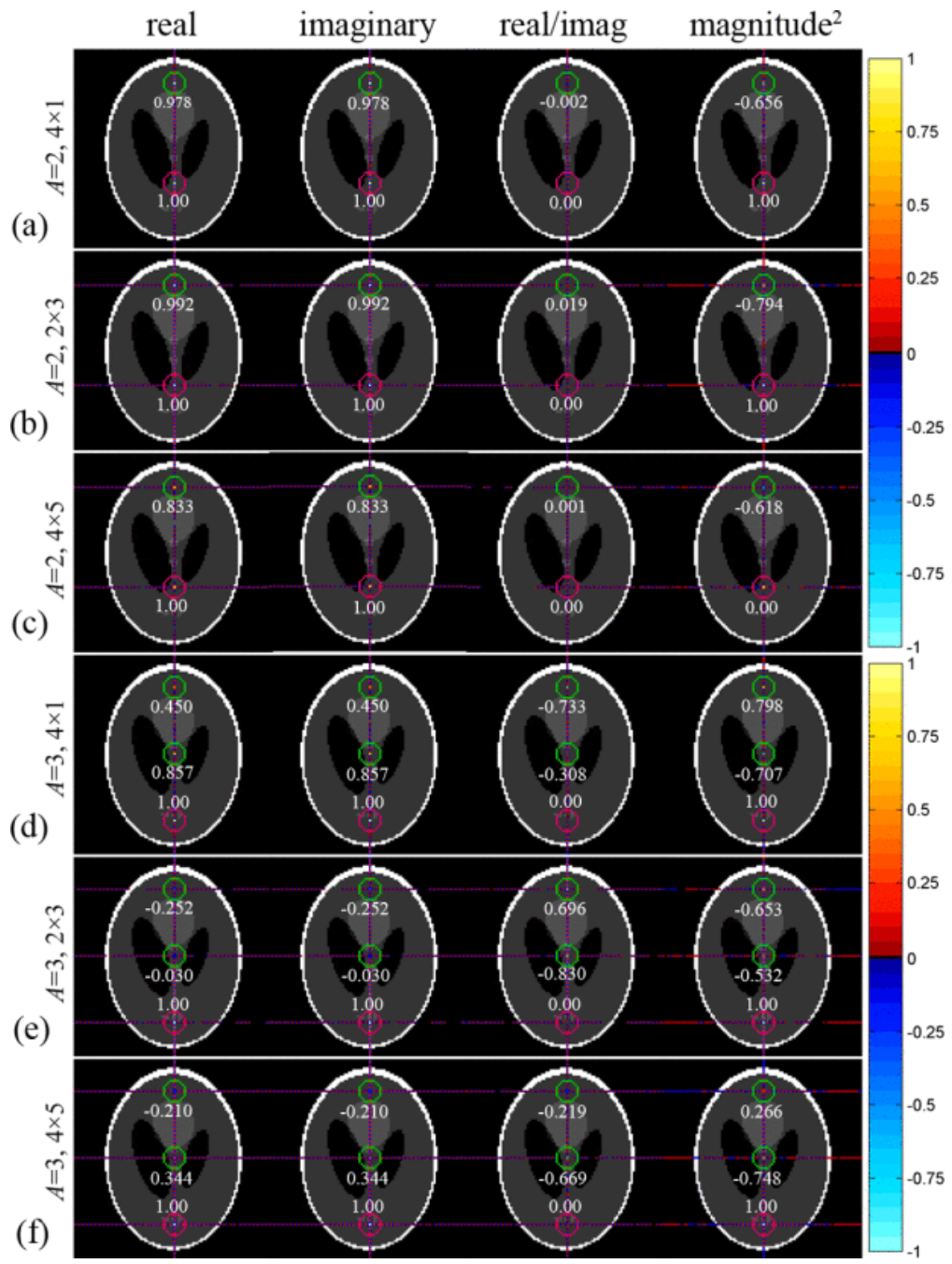

Fig. 4. Presented about a seed voxel in pink circle are theoretical correlations induced by GRAPPA reconstruction for an acceleration of $A=2$ with kernels of size (a) $4 \times 1$, (b) $2 \times 3$, and (c) $4 \times 5$, and for an acceleration of $A=3$ with kernels of size (d) $4 \times 1$, (e) $2 \times 3$, and (f) $4 \times 5$. Correlations are threshold to $10^{-7}$.

Presented in Fig. 4 are theoretical real, imaginary, real/imaginary and magnitudesquared correlations induced by the GRAPPA model about the seed voxel of interest (VOI) in the center of the pink circle for kernels of size $4 \times 1,2 \times 3$, and $4 \times 5$ with $A=2$ in

IEEE Transactions on Medical Imaging, Vol 33, No. 2 (February 2014): 495-503. DOI. This article is (C) Institute of Electrical and Electronics Engineers (IEEE) and permission has been granted for this version to appear in e-Publications@Marquette. Institute of Electrical and Electronics Engineers (IEEE) does not grant permission for this article to be further copied/distributed or hosted elsewhere without the express permission from Institute of Electrical and Electronics Engineers (IEEE). 
Fig. 4(a)-(c), and with $A=3$ in Fig. 4(d)-(f). Using a Shepp-Logan phantom for an underlay, the observed structures in Fig. 4 were accentuated by removing correlations outside of these areas, with a threshold close to zero, $\pm 10^{-7}$, applied to the remaining correlations. Due to spatial localization that results from using truncated convolution kernels, derived from the FT of the coil sensitivities, the strongest correlations in the real, imaginary and magnitude-squared images in Fig. 4 are between the VOI and previously aliased VOIs (aVOIs) in the center of the green circles. The induced correlations between each VOI and aVOI, stated adjacent to the respective voxel, appear to be (on average) inversely related to the size of the interpolation kernel. This is likely the result of a decreased relative weighting of each frequency measurement used in the interpolation. Similarly, the GRAPPA induced correlations are distributed among the $(A-1)$ aVOIs, and thus the strength of correlations induced with $A=3$ are lower than those with $A=2$. The vertical band of correlation in the column with the VOI and aVOI, as seen in all images of Fig. 4, results from interpolations in the PE direction, while the horizontal bands of correlation in the rows of the VOI and aVOIs, as seen in the images of Fig. 4 with 2-D kernels, results from interpolations in the frequency encoding direction. After an IFT, these bands exhibit an approximately sinc correlation with amplitudes and periods relative to the size of the rectangular kernels. The negative sign of the magnitude-squared correlation induced between the VOI and aVOI in Fig. 4 results from a combination of the magnitudesquared expected values and the covariance between the two voxels. The sign of the correlation between previously aliased voxels can therefore change from one voxel to the next, as will be seen in the experimental illustration, where this correlation is positive. 
NOT THE PUBLISHED VERSION; this is the author's final, peer-reviewed manuscript. The published version may be accessed by following the link in the citation at the bottom of the page.

(a)

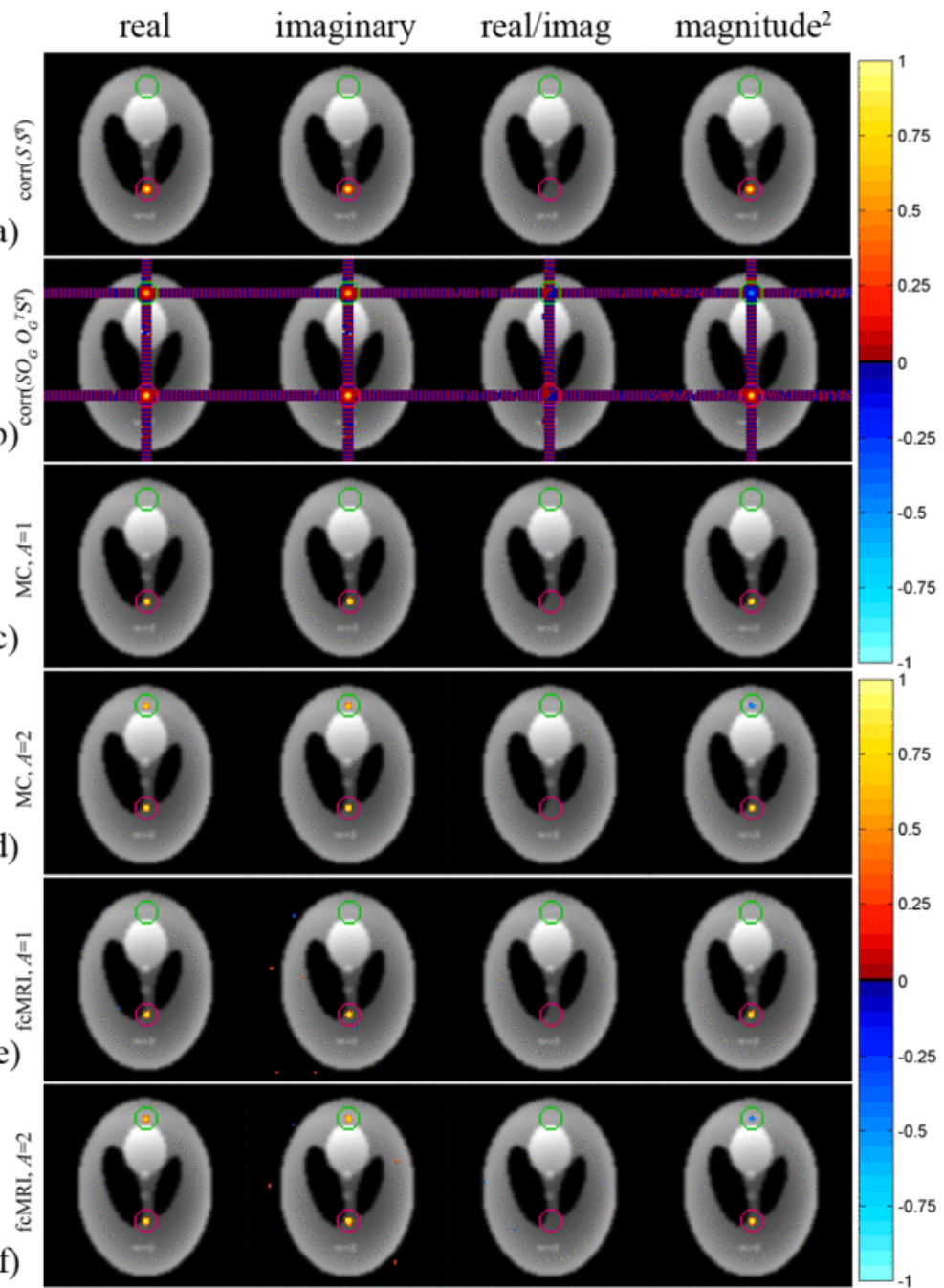

Fig. 5. Presented about a seed voxel in pink circle for a theoretical Shepp-Logan phantom are:

theoretical correlations induced by (a) spatial filtering, $S$, and (b) GRAPPA reconstruction with spatial filtering; simulated MC correlations for time series reconstructed with (c) $A=1$ (full FOV) with spatial filtering, and with (d) $A=2$ (GRAPPA reconstructed) with spatial filtering; simulated fcMRI time series Hamming band-pass filtered between 0.009 and $0.08 \mathrm{~Hz}$ for (e) $A=1$ (full FOV) with spatial filtering, and with (f) $A=2$ (GRAPPA reconstructed) with spatial filtering. Correlations in (a)-(b) threshold to $10^{-7}$ and correlations in (c)-(f) threshold to 0.35 .

Presented in Fig. 5(a) and (b) are the theoretical induced correlations from only the spatial filtering operator, $S$, and spatial filtering after a $4 \times 5$ GRAPPA image reconstruction with $A=2$. The correlations that exceed a threshold of $\pm 10^{-7}$, selected to be close to zero, are presented in Fig. 5(a) and (b), using full FOV and GRAPPA mean magnitude reconstructed images for underlays. As expected in Fig. 5(a), the process of smoothing induces a correlation between a voxel and its neighbors. Since Fig. 5(a) involves no pMRI reconstruction, $A=1$, there is no theoretical operator induced correlation between the VOI

IEEE Transactions on Medical Imaging, Vol 33, No. 2 (February 2014): 495-503. DOI. This article is (C) Institute of Electrical and Electronics Engineers (IEEE) and permission has been granted for this version to appear in e-Publications@Marquette. Institute of Electrical and Electronics Engineers (IEEE) does not grant permission for this article to be further copied/distributed or hosted elsewhere without the express permission from Institute of Electrical and Electronics Engineers (IEEE). 
in the pink circle and where an aVOI would be located in the green circle. By contrast in Fig. 5(b), the theoretical correlation induced by GRAPPA with a $4 \times 5$ kernel in Fig. 4(c) is accentuated with the addition of spatial filtering, as both the high correlations between the VOI and aVOI and the low bands of correlation are spread out and amplified.

In Fig. 5(c) and (d) are the correlations that exceed a liberal threshold of \pm 0.35 $(P \approx 0.05)$ [43], estimated directly from the full FOV and GRAPPA reconstructed MC simulated time series respectively. As both time series were generated with IID standard Gaussian noise, the correlations resulting from spatial filtering in the full FOV time series would only be expected to exist between the VOI and its immediate neighbors, shown in Fig. 5(c). However, in accordance with the theoretical correlations induced by GRAPPA in Fig. 4 and Fig. 5(b), there are notable positive real and imaginary correlations between the VOI and aVOI, as well a negative magnitude-squared correlation in Fig. 5(d). The negative sign of the theoretical magnitude-squared induced correlation in Fig. 5 again results from a combination of the magnitude-squared expected values and the covariance of the two voxels, and is relative to the magnitude signal strength of the aliased voxels.

The MC simulated fcMRI correlations that exceed a threshold of \pm 0.35 in the full FOV reconstructed data after Hamming band-pass filtering between 0.009 and $0.08 \mathrm{~Hz}$ are presented in Fig. 5(e). There is again no apparent structure between the VOI and any other voxel, but the imaginary correlations scattered throughout the image appear more significant after Hamming band-pass filtering in Fig. 5(e) by contrast to those in Fig. 5(c). However, the MC simulated correlation structure noted in the GRAPPA reconstructed time series in Fig. 5(d) is still apparent in Fig. 5(f) after being temporally filtered. As the data possessed no such correlation structure when it was generated, these correlations indicate that the GRAPPA reconstruction process induces correlations that reside in the frequency spectrum commonly associated with functional connectivity, and could thus have null hypothesis implications in a connectivity study marked by an increase in the false positive rate if no correlation is assumed between voxels.

\section{B. Experimental Results}

The averaged time series of a $2 \times 2$ seed region for the human subject was selected in the left posterior region of an axial slice of the brain such that the aVOI in a GRAPPA reconstructed image falls within the brain, and there is no correlation in full FOV data between the $2 \times 2$ seed region and where an aVOI would be located in full FOV data had sub-sampling been performed. The magnitude-squared operator induced correlations are presented for the full FOV and GRAPPA reconstructed data sets in Fig. 6(a) and (b), 
respectively. As with the theoretical operator induced correlations in Fig. 5(a) and (b), the operator induced correlations that exceed a threshold of $\pm 10^{-7}$ for the human subject only exhibit a local correlation between the VOI and its neighbors when spatial filtering is applied in the case of the full FOV data, and the GRAPPA induced correlations exhibit bands in the rows and columns of the VOI and the aVOI, with higher correlations in the neighborhood of the VOI and aVOI. The magnitude-squared experimental time series correlations that exceed a threshold of \pm 0.35 show that there is no correlation between the VOI and the aVOI in the full FOV reconstructed data in Fig. 6(c), by contrast to the GRAPPA reconstructed data in Fig. $6(\mathrm{~d})$ where there is a distinct $2 \times 2$ cluster of voxels in the aVOI correlated with the VOI.

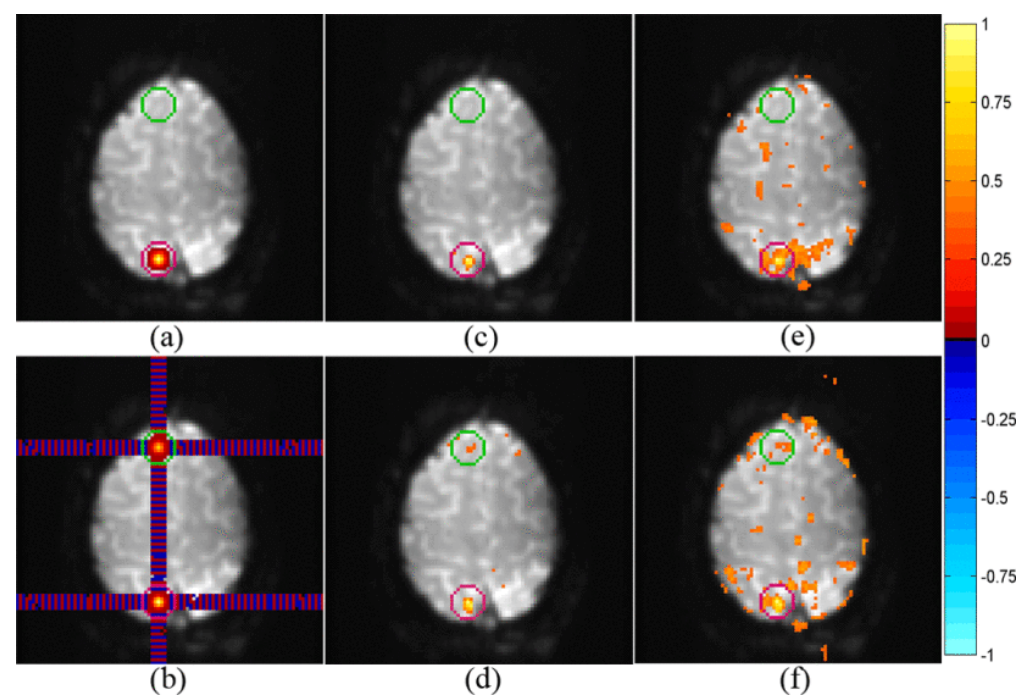

Fig. 6. Presented about a seed voxel in pink circle for a human subject are: magnitude-squared correlations induced by (a) spatial filtering, $S$, and (b) GRAPPA reconstruction with spatial filtering; experimentally estimated correlations for time series reconstructed with (c) $A=1$ (full FOV) with spatial filtering, and with (d) $A=2$ (GRAPPA reconstructed) with spatial filtering; experimentally estimated fcMRI time series Hamming band-pass filtered between 0.009 and $0.08 \mathrm{~Hz}$ for (e) $A=1$ (full FOV) with spatial filtering, and with (f) $A=2$ (GRAPPA reconstructed) with spatial filtering. Correlations in (a)-(b) threshold to $10^{-7}$ and correlations in (c)-(f) threshold to 0.35 .

After Hamming band-pass filtering the reconstructed time series to observe the fcMRI correlation coefficients in the human subject data, there is still no correlation between the VOI and aVOI in the case of the full FOV reconstructed data in Fig. 6(e), while there is again a distinct $2 \times 2$ cluster of voxels in the aVOI that are correlated with the VOI in Fig. 6(f) for the GRAPPA reconstructed data. The correlations between the VOI and aVOI in Fig. 6(f) are of note as they are not present in the full FOV data and they are still present after the Hamming band-pass filtering. This is consistent with the theoretical illustration in 
Fig. 5, and illustrates that the GRAPPA model induces correlations that are both statistically significant and reside in the frequency spectrum commonly associated with functional connectivity. A study that uses GRAPPA reconstructed fcMRI data could thus result in a false positive, assuming voxels to be correlated when they are not.

\section{SECTION V.}

\section{Discussion}

The work in this manuscript extends upon the AMMUST framework in ${ }^{14}$ to reconstruct sub-sampled data from an array of receiver coils using a real-valued isomorphic representation of the conventional complex-valued GRAPPA model. By representing the GRAPPA model in this mathematically equivalent way, it has been shown that the local correlation induced between neighboring spatial frequencies through the GRAPPA interpolation translates to a global correlation between multiple voxels in the full FOV reconstructed image. This correlation is most notable between voxels that were previously aliased because the GRAPPA interpolation effectively conducts a sensitivity encoding in $k$-space, with spatial localization performed by using a truncated convolution kernel that is derived from the FT of coil sensitivities. Despite the demand an isomorphism of this kind places on computational resources, it is only through a real-valued linear representation of the complex-valued GRAPPA image reconstruction process, such as the one presented here, that one can observe the artificial correlations induced between the real, the imaginary, between the real and imaginary, and between the square of the magnitude components of the reconstructed voxels. This is of particular importance given the increasing popularity of complex-valued fMRI and fcMRI studies. ${ }^{14,32-33,34}$

The implications of the GRAPPA reconstruction model were illustrated in both theoretical and experimental data sets, in which a comparison was made between data reconstructed with no sub-sampling and data sub-sampled by an acceleration factor of $A=$ 2 , reconstructed with the GRAPPA model. The framework developed in this manuscript has shown that the interpolation of missing spatial frequencies by the GRAPPA model induces a significant correlation between each reconstructed voxel and its previously aliased counterpart, with additional low correlations in the rows and columns of those voxels. As these correlations are of no biological origin, the degree to which they influence the correlations in functional connectivity studies was investigated by observing the correlations about a seed voxel with and without the band-pass filtering process undertaken in fcMRI studies. As a significant correlation between previously aliased voxels was noted in both the filtered and unfiltered GRAPPA reconstructed time series, but not in 
either of the full FOV reconstructed time series, it implies that the effects of the GRAPPA reconstruction process can have null hypothesis implications in a functional connectivity study in which no correlation is assumed between voxels. Depending on the placement of functionally connected networks within the brain, the PE direction, and the choice of $A$, the correlations induced between previously aliased voxels could either amplify or diminish the true correlation coefficients, thereby producing misleading results and conclusions. As such, care should be taken when investigating data acquired and reconstructed using pMRI models such as GRAPPA, and an analysis such as the one demonstrated in this manuscript should be performed. If an analysis of this kind cannot be performed, particular attention should be paid when correlations are noted between voxels spaced either $p_{y} / A$ or $p_{x} / A$ apart, depending on the direction of PE.

\section{References}

1J. S. Hyde, A. Jesmanowicz, W. Froncisz, J. B. Kneeland, T. M. Grist, N. F. Campagna, "Parallel image acquisition from noninteracting local coils", J. Magn. Reson., vol. 70, pp. 512-517, 1986.

${ }^{2}$ K. P. Pruessmann, M. Weiger, M. B. Scheidegger, P. Boesiger, "SENSE: Sensitivity encoding for fast MRI", Magn. Reson. Med., vol. 42, pp. 952-962, 1999.

${ }^{3}$ M. A. Griswold, P. M. Jakob, R. M. Heidemann, M. Nittka, V. Jellus, J. Wang, B. Kiefer, A. A. Haase, "Generalized autocalibrating partially parallel acquisitions (GRAPPA)", Magn. Reson. Med., vol. 47, pp. 1202-1210, 2002.

4I. P. Bruce, M. M. Karaman, D. B. Rowe, "A statistical examination of the SENSE reconstruction via an isomorphism representation", Magn. Reson. Imag., vol. 29, pp. 1267-1287, 2011.

5I. P. Bruce, M. M. Karaman, D. B. Rowe, "The SENSE-isomorphism theoretical image voxel estimation (SENSE-ITIVE) model for reconstruction and observing statistical properties of reconstruction operators", Magn. Reson. Imag., vol. 30, pp. 1143-1166, 2012.

6I. P. Bruce, D. B. Rowe, "Artificial correlations induced by SENSE and GRAPPA corrupt fcMRI conclusions", Proc. Int. Soc. Magn. Reson. Med., pp. 2229, 2013.

7I. P. Bruce, L. T. Muftuler, D. B. Rowe, "SENSE induced correlations are used to optimize RF coil design for specific fcMRI studies", Proc. Int. Soc. Magn. Reson. Med., pp. 2733, 2013.

${ }^{8}$ A. D. Hahn, A. S. Nencka, D. B. Rowe, "Improving robustness and reliability of phase-sensitive fMRI analysis using temporal off-resonance alignment of single-echo timeseries (TOAST)", Neuroimage, vol. 44, pp. 742-752, 2009.

${ }^{9}$ A. D. Hahn, D. B. Rowe, "Physiologic noise regression motion regression and TOAST dynamic field correction in complex-valued fMRI time series", Neuroimage, vol. 59, pp. 2231-2240, 2012.

${ }^{10}$ A. D. Hahn, A. S. Nencka, D. B. Rowe, " Enhancing the utility of complex-valued functional magnetic resonance imaging detection of neurobiological processes through postacquisition estimation and correction of dynamic $\backslash \$ B_{-}\{0\} \backslash \$$ errors and motion ", Hum. Brain Mapp., vol. 33, pp. 288306, 2012.

11P. Jezzard, R. S. Balaban, "Correction for geometric distortion in echo planar images from B0 inhomogeneity effects in MR images", Magn. Reson. Med., vol. 34, pp. 65-73, 1995. 
NOT THE PUBLISHED VERSION; this is the author's final, peer-reviewed manuscript. The published version may be accessed by following the link in the citation at the bottom of the page.

${ }^{12}$ D. B. Rowe, B. R. Logan, "A complex way to compute fMRI activation", Neuroimage, vol. 23, pp. 10781092, 2004.

${ }^{13} \mathrm{D}$. B. Rowe, B. R. Logan, "Complex fMRI analysis with unrestricted phase is equivalent to a magnitudeonly model", Neuroimage, vol. 24, pp. 603-606, 2005.

${ }^{14}$ D. B. Rowe, "Modeling both the magnitude and phase of complex-valued fMRI data", Neuroimage, vol. 25, pp. 1310-1324, 2005.

15D. B. Rowe, "Magnitude and phase signal detection in complex-valued fMRI data", Magn. Reson. Med., vol. 62, pp. 1356-1357, 2009.

16S. K. Arja, Z. Feng, Z. Chen, A. Caprihan, K. A. Kiehl, T. Adali, V. D. Calhoun, "Changes in fMRI magnitude data and phase data observed in block-design and event-related tasks", Neuroimage, vol. 49, pp. 3149-3160, 2010.

17M. M. Karaman, I. P. Bruce, D. B. Rowe, "Temporal processing of fMRI data induces functional correlations and potentially alters functional activations", Proc. Int. Soc. Magn. Reson. Med., pp. $2232,2013$.

${ }^{18}$ A. S. Nencka, A. D. Hahn, D. B. Rowe, " A mathematical model for understanding statistical effects of $\backslash \$\{\mathrm{rm} \mathrm{k}\} \backslash \$$-space (AMMUST- $\backslash \$\{\mathrm{rm} \mathrm{k}\} \backslash \$$ ) preprocessing on observed voxel measurements in fcMRI and fMRI ", J. Neurosci. Meth., vol. 181, pp. 268-282, 2009.

${ }^{19}$ D. B. Rowe, A. S. Nencka, R. G. Hoffmann, "Signal and noise of Fourier reconstructed fMRI data", J. Neurosci. Meth., vol. 159, pp. 361-369, 2007.

20S. C. Strother, "Evaluating fMRI preprocessing pipelines", IEEE Eng. Med. Biol. Mag., vol. 25, pp. 27-41, 2006.

21R. L. Barry, S. C. Strother, J. C. Gore, "Complex and magnitude-only preprocessing of 2-D and 3D BOLD fMRI data at 7 T", Magn. Reson. Med., vol. 3, pp. 867-871, 2011.

${ }^{22}$ M. A. Griswold, M. Blaimer, F. Breuer, R. M. Heidemann, M. Mueller, P. M. Jakob, "Parallel magnetic resonance imaging using the GRAPPA operator formalism", Magn. Reson. Med., vol. 54, pp. 15531556, 2005.

23P. M. Robson, A. K. Grant, A. J. Madhuranthakam, R. Lattanzi, D. K. Sodickson, C. A. McKenzie, " Comprehensive quantification of signal-to-noise ratio and $\backslash \$\{\mathrm{rm} \mathrm{g}\} \backslash \$$-factor for image-based and $\backslash \$\{\mathrm{rm} \mathrm{k}\} \backslash \$$-space-based parallel imaging reconstructions ", Magn. Reson. Med., vol. 60, pp. 895-907, 2008.

${ }^{24}$ B. Biswal, F. Z. Yetkin, V. M. Haughton, J. S. Hyde, "Functional connectivity in the motor cortex of resting human brain echo-planar MRI", Magn. Reson. Med., vol. 34, pp. 537-541, 1995.

25M. A. Griswold, " Advanced $\backslash \$\{\mathrm{rm} \mathrm{k}\} \backslash \$$-space techniques ", Proc. 2nd Int. Workshop Parallel Imag., pp. 16-18, 2004.

26Z. Wang, J. Wang, J. A. Detre, "Improved data reconstruction method for GRAPPA", Magn. Reson. Med., vol. 54, pp. 738-742, 2005.

27M. A. Griswold, F. Breuer, M. Blaimer, S. Kannengiesser, R. M. Heidemann, M. Mueller, M. Nittka, V. Jellus, B. Kiefer, P. M. Jakob, "Autocalibrated coil sensitivity estimation for parallel imaging", NMR Biomed., vol. 19, pp. 316-324, 2006.

${ }^{28}$ A. C. S. Brau, P. J. Beatty, S. Skare, R. Bammer, "Comparison of reconstruction accuracy and efficiency among autocalibrating data-driven parallel imaging methods", Magn. Reson. Med., vol. 59, pp. 382-395, 2008

IEEE Transactions on Medical Imaging, Vol 33, No. 2 (February 2014): 495-503. DOI. This article is (C) Institute of Electrical and Electronics Engineers (IEEE) and permission has been granted for this version to appear in e-Publications@Marquette. Institute of Electrical and Electronics Engineers (IEEE) does not grant permission for this article to be further copied/distributed or hosted elsewhere without the express permission from Institute of Electrical and Electronics Engineers (IEEE). 
${ }^{29}$ D. J. Larkman, R. G. Nunes, "Parallel magnetic resonance imaging", Phys Med. Biol., vol. 52, pp. 15-55, 2007.

30F. Breuer, S. A. R. Kannengiesser, M. Blaimer, N. Seiberlich, P. M. Jakob, M. A. Griswold, " General formulation of quantitative $\backslash \$\{\mathrm{rm}$ g $\} \backslash \$$-factor calculation in GRAPPA reconstruction ", Magn. Reson. Med., vol. 62, pp. 739-746, 2009.

31J. Park, Q. Zhang, V. Jellus, O. Simonetti, D. Li, " Artifact and noise suppression in GRAPPA imaging using improved $\backslash \$\{\mathrm{rm} \mathrm{k}\} \backslash \$$-Space coil calibration and variable density sampling ", Magn. Reson. Med., vol. 53, pp. 186-193, 2005.

32R. S. Menon, "Postacquisition suppression of large-vessel BOLD signals in sigh-resolution fMRI", Magn. Reson. Med., vol. 47, pp. 1-9, 2002.

${ }^{33}$ A. S. Nencka, D. B. Rowe, "Reducing the unwanted draining vein BOLD contribution in fMRI with statistical post-processing methods", Neuroimage, vol. 37, pp. 177-188, 2007.

34J. R. Reichenbach, "The future of susceptibility contrast for assessment of anatomy and function", Neuroimage, vol. 62, pp. 1311-1315, 2012.

35J. Miao, S. Narayan, D. Huo, D. L. Wilson, " Modeling non-stationarity of kernel weights for $\backslash \$\{\mathrm{rm} \mathrm{k}\} \backslash \$$ space reconstruction in partially parallel imaging ", Med. Phys., vol. 38, no. 8, pp. 4760-4773, 2011.

36S. Bauer, M. Markl, M. Honal, B. A. Jung, "The effect of reconstruction and acquisition parameters for GRAPPA-based parallel imaging on the image quality", Magn. Reson. Med., vol. 66, no. 2, pp. 402409, 2011.

37R. M. Birn, J. B. Diamond, M. A. Smith, P. A. Bendettini, "Separating respiratory-variation-related fluctuations from neuronal-activity-related fluctuations in fMRI", Neuroimage, vol. 31, pp. 15361548, 2006.

38J. L. Vincent, A. Z. Snyder, M. D. Fox, J. B. Shannon, L. R. Andrews, M. E. Raichle, R. L. Buckmer, "Coherent spontaneous activity identifies a hippocampal-parietal memory network", J. Neurophysiol., vol. 96, pp. 3517-3531, 2006.

${ }^{39}$ S. J. Li, Z. Li, G. Wu, M. Zhang, M. Franczak, P. G. Antuono, "Alzheimer disease: Evaluation of a functional MR imaging index as a marker", Neuroradiology, vol. 225, no. 1, pp. 253-259, 2002.

${ }^{40}$ A. S. Nencka, A. D. Hahn, D. B. Rowe, "The use of three navigator echoes in cartesian EPI reconstruction reduces nyquist ghosting", Proc. Int. Soc. Magn. Reson. Med., pp. 3032, 2008.

41D. B. Rowe, A. S. Nencka, " Induced correlations in FMRI magnitude data from $\backslash \$\{\mathrm{rm} \mathrm{k}\} \backslash \$$-space preprocessing ", Proc. Int. Soc. Magn. Reson. Med., pp. 1721, 2009.

${ }^{42}$ K. J. Friston, P. Jezzard, R. Turner, "Analysis of functional MRI time-series", Hum. Brain Mapp., vol. 1, pp. 153-171, 1994.

43D. Cordes, V. M. Haughton, K. Arfanakis, G. J. Wendt, P. A. Turski, C. H. Moritz, M. A. Quigley, M. E. Meyerand, "Mapping functionally related regions of brain with functional connectivity MR imaging", Am. J. Neuroradiol., vol. 21, pp. 1636-1644, 2000.

${ }^{44}$ M. E. Raichle, A. M. MacLeod, A. Z. Snyder, W. J. Powers, D. A. Gusnard, G. L. Shulman, "A default mode of brain function", Proc. Nat. Acad. Sci. USA, vol. 98, no. 2, pp. 676-682, 2001.

IEEE Transactions on Medical Imaging, Vol 33, No. 2 (February 2014): 495-503. DOI. This article is (C) Institute of Electrical and Electronics Engineers (IEEE) and permission has been granted for this version to appear in e-Publications@Marquette. Institute of Electrical and Electronics Engineers (IEEE) does not grant permission for this article to be further copied/distributed or hosted elsewhere without the express permission from Institute of Electrical and Electronics Engineers (IEEE). 